\title{
Bioprosthetic Aortic Scallop Intentional Laceration to prevent latrogenic Coronary Artery obstruction (BASILICA) during transcatheter aortic valve-in-valve implantation with bioprosthetic valve fracturing via the transcaval access
}

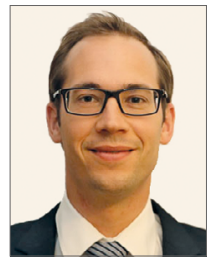

Jonas Lanz ${ }^{1}$, MD, MSc; Thomas Pilgrim¹, MD; Adam B. Greenbaum², MD; Stephan Windecker ${ }^{1 *}$, MD

1. Department of Cardiology, Swiss Cardiovascular Center, University Hospital Bern, Bern, Switzerland;

2. Center for Structural Heart Disease, Division of Cardiology, Henry Ford Hospital, Detroit, MI, USA

This paper also includes supplementary data published online at: http://www.pcronline.com/eurointervention/141st_issue/157

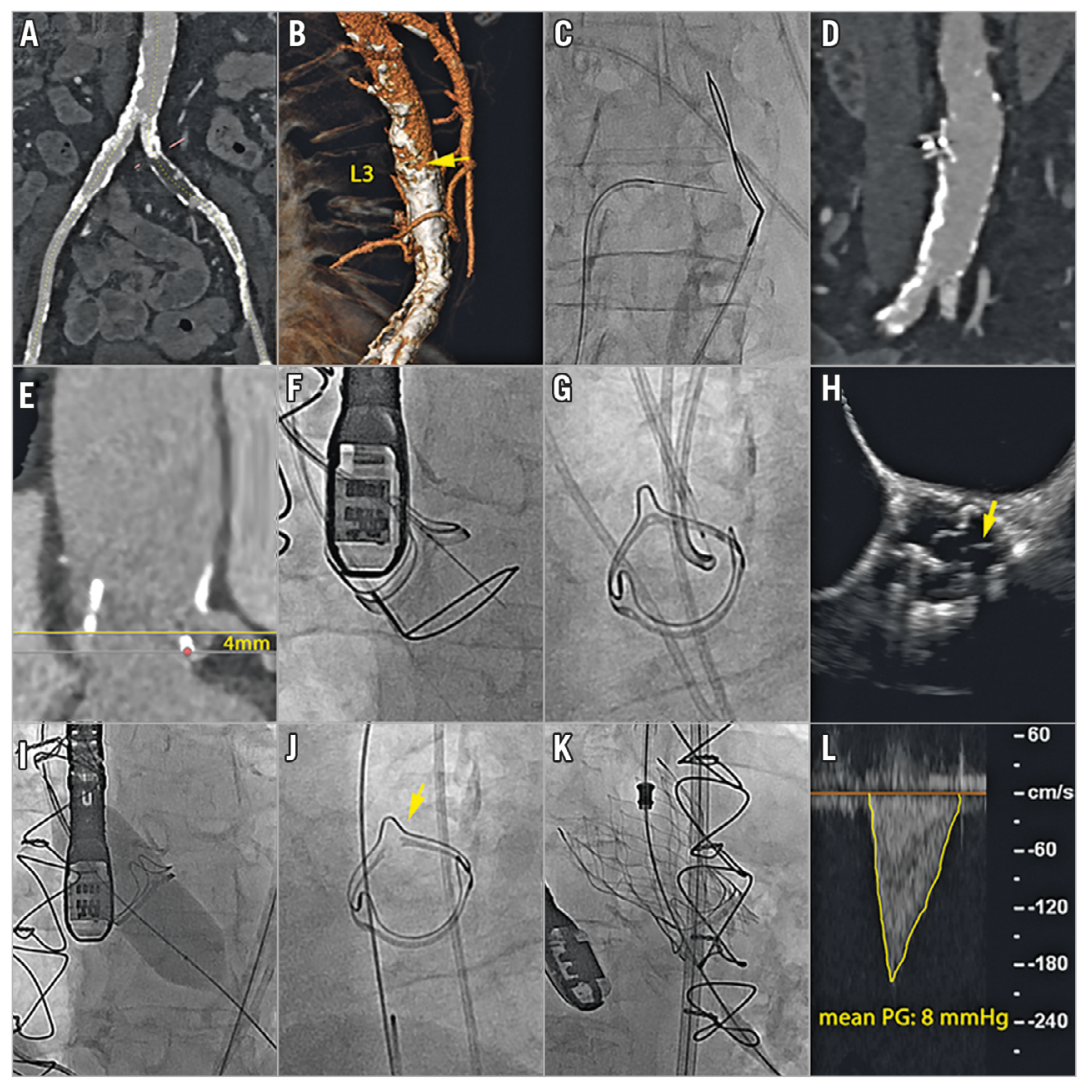

Figure 1. Threefold challenge of transcatheter aortic valve-in-valve implantation. A-D) Access: unsuitable peripheral arterial access (A), favourable aortic target zone (B, arrow) for caval-aortic crossing (C) with an Astato XS 20 guidewire (Asahi Intecc Co., Ltd., Aichi, Japan) at the level of lumbar vertebra 3 (L3), closure of the aortic entry site with a 10/8 mm AMPLATZER ${ }^{\mathrm{TM}}$ Duct Occluder (ADO 1) (St. Jude Medical, St. Paul, MN, USA). E-H) Risk of coronary artery obstruction: low (4 mm) take-off of the left coronary ostium (E), Bioprosthetic Aortic Scallop Intentional Laceration to prevent Iatrogenic Coronary Artery obstruction (BASILICA) encompassing perforation of the surgical bioprosthetic scallop $(F)$ with an Astato XS 20 wire, creation of a loop and laceration by electrocautery application $(G)$, achieving a split of the bioprosthetic scallop (H, arrow) in proximity to the left coronary ostium. I-L) Undersized surgical bioprosthesis: inflation of a $22 \mathrm{~mm}$ high-pressure balloon (I) causing fracture of the ring of the PERIMOUNT Magna 21 mm surgical bioprosthesis without haemodynamic compromise (J), adequate expansion of the self-expanding $23 \mathrm{~mm}$ CoreValve ${ }^{\circledR}$ Evolut $^{\mathrm{TM}} \mathrm{R}$ (Medtronic, Minneapolis, MN, USA) transcatheter prosthesis $(K)$ as reflected by a low post-procedural mean transvalvular pressure gradient $(P G)$ of $8 \mathrm{mmHg}(\mathrm{L})$.

*Corresponding author: Department of Cardiology, Bern University Hospital, University of Bern, Freiburgstrasse 8-10, 3010 Bern, Switzerland.E-mail: stephan.windecker@insel.ch 
A 75-year-old female patient with a history of surgical aortic valve replacement (SAVR) in 2007 developed progressive dyspnoea on exertion (New York Heart Association Class III-IV). Echocardiography revealed severely reduced left ventricular ejection fraction and degeneration with severe stenosis of the $21 \mathrm{~mm}$ Carpentier-Edwards PERIMOUNT Magna aortic bioprosthesis (Edwards Lifesciences, Irvine, CA, USA). The Heart Team advised the patient against repeat SAVR. With a view to transcatheter valve-in-valve (ViV) implantation, three procedural challenges were encountered related to access site, risk of coronary artery obstruction and risk of persistently elevated transvalvular gradient due to underexpansion of the $\mathrm{ViV}$ prosthesis. Figure 1 illustrates how all three obstacles were successfully overcome by choosing the transcaval access ${ }^{1}$ (Figure 1A-Figure 1D, Moving image 1, Moving image 2A, Moving image 2B), performing a Bioprosthetic Aortic Scallop Intentional Laceration to prevent Iatrogenic Coronary Artery obstruction (BASILICA) ${ }^{2}$ (Figure 1E-Figure 1H, Moving image 3-Moving image 5) and inducing an intentional fracture of the ring of the surgical bioprosthesis before implantation of the transcatheter valve ${ }^{3}$ (Figure 1I-Figure 1L).

\section{Conflict of interest statement}

S. Windecker has received research grants to the institution from Abbott, Amgen, Biotronik, Boston Scientific, St. Jude Medical, Terumo and Bayer. T. Pilgrim has received research grants to the institution from Biotronik, Symetis, and Edwards Lifesciences, and speaker fees from Boston Scientific. A. Greenbaum is a proctor for Edwards Lifesciences and Medtronic, and has patents pending on equipment related to the transcaval access and closure that have been assigned to his former employer, the Henry Ford Hospital, Detroit, USA. J. Lanz has no conflicts of interest to declare.

\section{References}

1. Greenbaum AB, Babaliaros VC, Chen MY, Stine AM, Rogers T, O’Neill WW, Paone G, Thourani VH, Muhammad KI, Leonardi RA, Ramee S, Troendle JF, Lederman RJ. Transcaval
Access and Closure for Transcatheter Aortic Valve Replacement: A Prospective Investigation. J Am Coll Cardiol. 2017;69:511-21.

2. Khan JM, Dvir D, Greenbaum AB, Babaliaros VC, Rogers T, Aldea G, Reisman M, Mackensen GB, Eng MHK, Paone G, Wang DD, Guyton RA, Devireddy CM, Schenke WH, Lederman RJ. Transcatheter Laceration of Aortic Leaflets to Prevent Coronary Obstruction During Transcatheter Aortic Valve Replacement: Concept to First-in-Human. JACC Cardiovasc Interv. 2018;11:677-89.

3. Chhatriwalla AK, Allen KB, Saxon JT, Cohen DJ, Aggarwal S, Hart AJ, Baron SJ, Dvir D, Borkon AM. Bioprosthetic Valve Fracture Improves the Hemodynamic Results of Valve-in-Valve Transcatheter Aortic Valve Replacement. Circ Cardiovasc Interv. $2017 \mathrm{Jul} ; 10(7)$.

\section{Supplementary data}

Moving image 1. Caval-aortic crossing with Astato XS 20 wire during electrocautery application - anterior-posterior and left lateral view.

Moving image 2A. $10 / 8 \mathrm{~mm}$ AMPLATZER Duct Occluder (ADO-1) implantation.

Moving image 2B. Final abdominal aortic angiography showing complete closure of aortic entry site.

Moving image 3. Perforation of the surgical bioprosthetic scallop with an Astato XS 20 wire during electrocautery application anterior-posterior and left-anterior oblique view.

Moving image 4. Intentional laceration (splitting) of the bioprosthetic aortic scallop by electrocautery application - anterior-posterior and left lateral view.

Moving image 5. Split bioprosthetic scallop - with and without colour Doppler illustrating proximity to the left coronary ostium.

The supplementary data are published online at:

http://www.pcronline.com/

eurointervention/141st_issue/157 